\title{
Parietal foramina with cleidocranial dysplasia is caused by mutation in MSX2
}

\author{
Sixto Garcia-Miñaur ${ }^{1,6}$, Lampros A Mavrogiannis ${ }^{2,6}$, Sahan V Rannan-Eliya ${ }^{2}$, \\ Michael A Hendry ${ }^{3}$, William A Liston ${ }^{4}$, Mary EM Porteous ${ }^{1}$ and Andrew OM Wilkie ${ }^{\star, 2,5}$
}

${ }^{1}$ South East Scotland Genetic Service, Western General Hospital, Edinburgh, UK; ${ }^{2}$ Weatherall Institute of Molecular Medicine, University of Oxford, Oxford, UK; ${ }^{3}$ Paediatric Radiology Department, Royal Hospital for Sick Children, Edinburgh, UK; ${ }^{4}$ The Simpson Centre for Reproductive Health, Royal Infirmary of Edinburgh, Edinburgh, UK;

${ }^{5}$ Nuffield Department of Clinical Laboratory Sciences, University of Oxford, Oxford, UK

The combination of skull defects in the form of enlarged parietal foramina (PFM) and deficient ossification of the clavicles is known as parietal foramina with cleidocranial dysplasia (PFMCCD). It is considered to be distinct from classical cleidocranial dysplasia (CCD) and is listed as a separate OMIM entry (168550). So far, only two families have been reported and the molecular basis of the disorder is unknown. We present a third family with PFMCCD, comprising four affected individuals in three generations, and demonstrate that a heterozygous tetranucleotide duplication in the MSX2 homeobox gene (505_508dupATTG) segregates with the phenotype. PFMCCD is indeed aetiologically distinct from CCD, which is caused by mutations in the RUNX2 gene, but allelic with isolated PFM, in which MSX2 mutations were previously identified. Our observations highlight the role of $M S X 2$ in clavicular development and the importance of radiological examination of the clavicles in subjects with PFM.

European Journal of Human Genetics (2003) 11, 892-895. doi:10.1038/sj.ejhg.5201062

Keywords: parietal foramina; clavicular hypoplasia; cleidocranial dysplasia; MSX2; ALX4; RUNX2

\section{Introduction}

Parietal foramina (PFM) are symmetrical oval defects of the parietal bones that reflect deficient or delayed ossification around the parietal notch during skull development; a fibrous membrane covers the openings that tend to ossify naturally with age. PFM may be encountered as an isolated finding, usually inherited as an autosomal-dominant trait, or in a syndromic context, such as the proximal $11 \mathrm{p}$ deletion syndrome ${ }^{1}$ or Saethre-Chotzen syndrome. ${ }^{2}$ Isolated, nonsyndromic PFM are caused by loss-of-function mutations in the $\mathrm{MSX2}^{3,4}$ (PFM1; OMIM 123101) and the $A L X 4^{5,6}$ (PFM2; OMIM 605420) homeobox genes.

\footnotetext{
${ }^{*}$ Correspondence: Prof AOM Wilkie, Weatherall Institute of Molecular Medicine, John Radcliffe Hospital, Headington, Oxford OX3 9DS, UK. Tel: + 441865222 619; Fax: + 441865222 500; E-mail: awilkie@molbiol.ox.ac.uk

${ }^{6}$ The first two authors contributed equally to this work Received 14 February 2003; revised 26 May 2003
}

Cleidocranial dysplasia (CCD; OMIM 119600) is the major skeletal disorder associated with congenital clavicular hypoplasia. It is characterised by a generalised delay in ossification of the skull, hypoplastic or absent clavicles, short stature and abnormal dentition. Clavicular agenesis is rare, whereas hypoplasia of the lateral ends is typical. Occasionally, manifestations reminiscent of clavicular pseudoarthrosis (a central gap with bone replacement by fibrous tissue), or absence of the medial end with the lateral end present, are seen. ${ }^{7}$ Over 60 different heterozygous intragenic mutations of the RUNX2 gene, encoding a runt domain transcription factor (CBFA1), have been identified in CCD. ${ }^{8}$

The occurrence of both PFM and clavicular hypoplasia is known as parietal foramina with cleidocranial dysplasia (PFMCCD). It is listed as a separate entity in both OMIM (168550) and the London Dysmorphology Database. ${ }^{9}$ To our knowledge, only two families with PFMCCD have been reported, both compatible with an autosomal-dominant 
mode of inheritance. The original family included an affected mother and son. ${ }^{10}$ The 4 -year-old boy showed large paired parietal defects and abnormal clavicles, with hypoplasia of the lateral (acromial) ends and unusually thick medial (sternal) ends; the mother had bilateral small parietal defects, and her clavicles were unusually straight and thick. In a second family, ${ }^{11,12}$ a father and his 6-month-old son exhibited PFM, hypoplastic, laterally tapering clavicles and macrocephaly; in addition, the son had unilateral microtia and an occipital dermoid cyst.

Here we describe a new three-generation family segregating PFMCCD in four affected individuals. A heterozygous frameshift mutation in MSX2 was detected in affected family members. This confirms that PFMCCD is distinct from CCD, and identifies a causative genetic change for the first time. We discuss the association of cranial and clavicular ossification defects in relation to the evolutionary origin and developmental derivation of these structures.

\section{Methods}

The coding region of MSX2 (reference sequences: GenBank L22498, L22499) was amplified from genomic DNA using the primer pairs described in Wilkie et al, ${ }^{3}$ with annealing temperatures of $56^{\circ} \mathrm{C}$ for exon 1 and $60^{\circ} \mathrm{C}$ for exon 2 . The fragments were screened for variants using denaturing high-performance liquid chromatography (DHPLC) on the WAVE DNA Fragment Analyser System (Transgenomic Ltd, Crewe, Cheshire, UK). The analyses were performed at 65 and $68^{\circ} \mathrm{C}$ for exon 1 and at 62 and $63^{\circ} \mathrm{C}$ for exon 2 , and included heterozygous controls for the polymorphisms c. $17 \mathrm{C}>\mathrm{G}$ and $386 \mathrm{C}>\mathrm{T}$. PCR products and cloned fragments were sequenced by means of the BigDye terminator kit (Applied Biosystems, Foster City, CA, USA) and the reactions were analysed on the ABI PRISM 3100 sequencer (Applied Biosystems). To resolve the mutant allele, the exon 2 PCR product was ligated into pGEM-T Easy vector (Promega Corporation, Madison, WI, USA) and mutant clones were selected after EcoRI and BsrDI digestion (New England Biolabs (UK) Ltd, Hitchin, Hertfordshire, UK). The 505_508dupATTG change was verified by digestion of the 496 bp exon 2 PCR product with BsrDI: the mutation abolishes a unique cleavage site that normally yields fragments of 163 and $333 \mathrm{bp}$.

Segregation of RUNX2 in the pedigree was tested using two newly developed simple sequence repeat polymorphisms (GenBank AL161907). The primer pair 5'-GCATTT CATTCTACCCCGAATTGC-3' and 5'-GCAGGGGCAACAA GAGCGAAC-3' amplifies a $(\mathrm{CA})_{n}$ repeat within intron 6 (according to exon nomenclature of Otto et $\mathrm{al}^{8}$ ) of RUNX2 and primer pair 5'-TGGAAGCCTGAGTAATTCCTTGC-3' and 5'-AGCCTGGGGTGATTCTCTCTTTC-3' amplifies an
$(\mathrm{AAAG})_{n}$ repeat located $14 \mathrm{~kb}$ downstream of the gene. The heterozygosities of these loci were 79\% (104 chromosomes) for the $(\mathrm{CA})_{n}$ repeat and $82 \%$ (152 chromosomes) for the $(\mathrm{AAAG})_{n}$ repeat. The $A L X 4$ gene was screened by DHPLC using the primers described previously. ${ }^{6}$

\section{Clinical report}

The family pedigree is summarised in Figure 1a. PFM were first noticed in the proband (III-1) at the age of 5 years. The diagnosis of PFMCCD was established at the age of 18 years, following incidental radiological examination because of a head injury. His father (II-1) had palpable PFM and reported that a chest radiograph taken as part of a routine Army medical examination had revealed short clavicles. The paternal grandmother (I-1) is reputed to have sloping shoulders but was not available for assessment. The proband has three children, two of whom share similar defects; both also have broad foreheads with frontal bossing, but their head circumferences are within normal limits. The elder son (IV-1) presented with typical PFM, whereas the younger son (IV-3) had a $4 \mathrm{~cm}$-wide posterior fontanelle and a $5 \mathrm{~cm}$-long anterior fontanelle at the age of 3 months. There were no dental problems in any of the affected members.

Radiological examination confirmed the skull and clavicular ossification defects in the three individuals examined (Figure 2). The 31-year-old proband (III-1) showed bilateral PFM, while his clavicles were straight, short and rather undertubulated, with irregular medial, and tapering lateral ends. His 62-year-old father (II-1) had small bilateral PFM and his clavicles were short, broad and coarse, with tapering lateral ends. The 4-year-old elder son

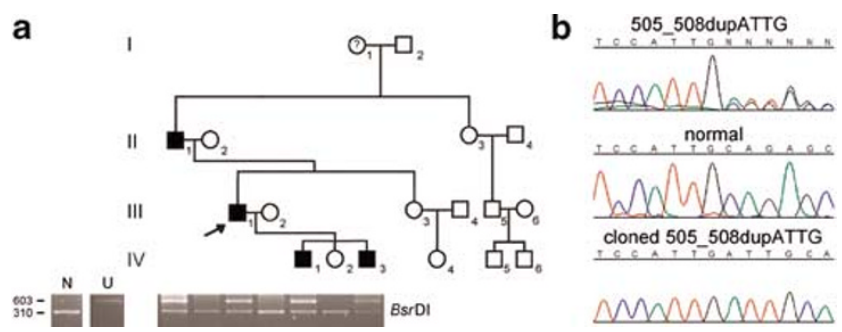

Figure 1 A frameshift mutation in MSX2 segregates with PFMCCD in the three-generation family. (a) Pedigree showing affected individuals (filled symbols); the proband is indicated by an arrow. Segregation of a BsrDI digest of MSX2 exon 2 is shown below the corresponding pedigree symbols; $N$, digest of normal control genomic DNA; $U$, undigested PCR product. The paternal grandmother (I-1) was not available for examination. (b) Sequence traces (forward direction) of MSX2 exon 2 from III-1 genomic DNA (top), normal control (middle), and cloned mutant allele from III-1 (bottom). 

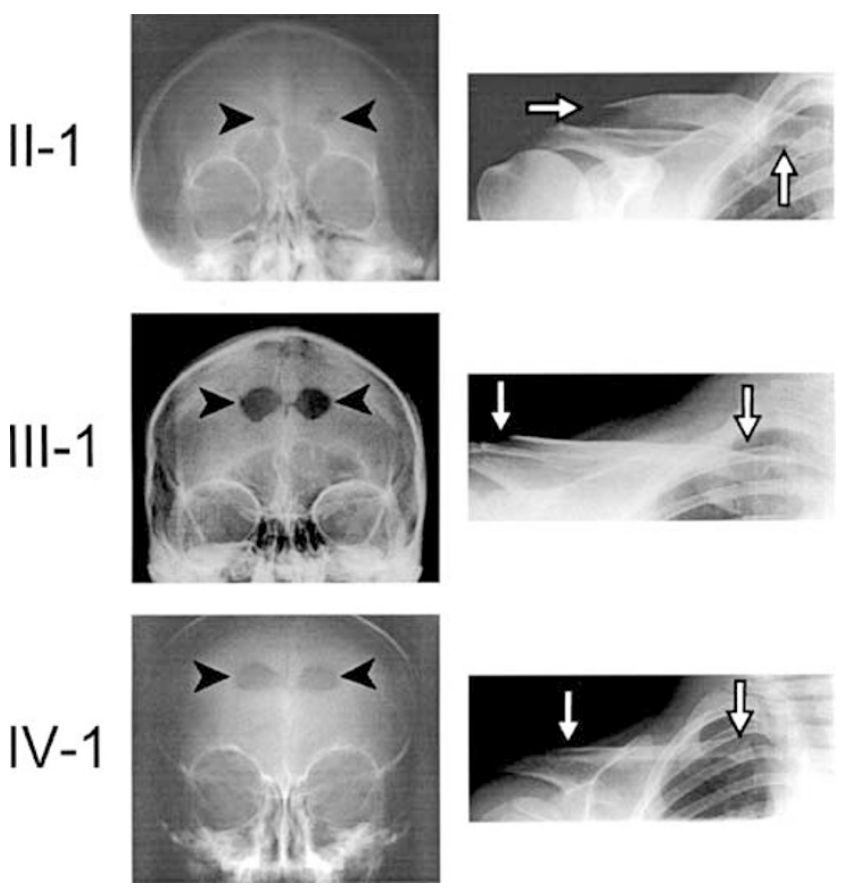

Figure 2 Skull and chest radiographs of II- 1 aged 63 years (top), the proband III-1 aged 32 years (middle) and IV-1 (skull at age 3.5 years; chest at age 5 years) (bottom). PFM are indicated by black arrowheads; these skull defects tend to decrease in size with age and are not so evident in II-1. Note abnormal modelling of clavicles (white arrows): in II1 , short, broad and coarse appearance with tapering lateral end (left arrow) and prominent rhomboid fossa (right arrow), a normal variant; narrow straight clavicles with tapering ends in III-1; short straight clavicle with tapering lateral end in IV-1.

(IV-1) showed bilateral PFM and, similarly, his clavicles were short with rounded lateral ends.

Following DHPLC analysis and sequencing, the heterozygous duplication of a tetranucleotide (505_508dupATTG), located in the second exon of the MSX2 gene, was identified in III-1 (Figure 1B). This mutation abolishes a $B s r$ DI restriction site and was shown to be present, in the heterozygous state, in all four affected individuals, but absent in IV-2, an unaffected individual at 50\% prior risk (Figure 1a). The duplication causes a frameshift within the homeobox of MSX2; in the new frame, a termination codon is predicted some distance downstream (A170fsX245). This may either destabilise the transcript and/or the protein, or result in an aberrant product.

We excluded a coexisting mutation of $A L X 4$ by DHPLC analysis of the coding region in III-1, and showed, for two simple sequence repeats physically linked to RUNX2, that IV-1 inherited the allele originating from the proband's unaffected mother II-2 (not illustrated). This rules out the coexisting segregation of an RUNX2 mutation with the phenotype.

\section{Discussion}

The association of PFM and deficient ossification of the clavicles has been referred to as PFMCCD. Although the molecular bases of both isolated PFM and CCD have been delineated, no molecular analysis has previously been undertaken on this combined entity. Analysis of PFM has identified heterozygous missense and nonsense mutations as well as deletions in two genes, MSX2 $2^{3,4}$ and $A L X 4^{5,6}$ both of which encode homedomain transcription factors. To date, six intragenic mutations and one complete deletion of MSX2 have been described in patients or families with PFM. ${ }^{3,4}$ The deletion caused a phenotype similar to that associated with the intragenic mutations, suggesting haploinsufficiency of MSX2 as the pathophysiological mechanism. This was corroborated by demonstrating loss of target DNA binding in the case of two changes (one missense, one 2-amino-acid deletion) within the homeodomain. ${ }^{3}$

In this work, we describe a new family with PFMCCD, the third to be reported, and identify a heterozygous frameshift mutation in MSX2 as the causative change. The affected individuals in this family exhibited classical PFM and short abnormal clavicles with tapering lateral ends. They had mild craniofacial dysmorphism with a broad forehead and central bossing, which was more evident in the children. There were no scalp defects. The MSX2 mutation in this family provides the first evidence that development of the clavicles, as well as the skull, is particularly sensitive to MSX2 protein dosage. The mutation identified is predicted to cause a frameshift at the 29th residue of the homeodomain, with a new stop codon located a further 75 triplets downstream. The mutant protein is likely to be nonfunctional, as it does not contain either the DNA-binding helix III of the homeodomain or its supporting helix II; additionally, the potential product in the new frame shares no similarity with other proteins. Even if transcription and translation take place, haploinsufficiency of MSX2 is likely, although a dominant-negative effect cannot, in principle, be excluded. Alternatively, the mutant transcript and/or protein may be unstable, also leading to haploinsufficiency.

Why is the clavicle, as well as the skull vault, particularly sensitive to MSX2 dosage? Evolutionary origins could provide clues. The flat bones of the skull are dermal bones that ossify intramembranously - they are traced back to the extensive exoskeletal armour that encased many early vertebrates. ${ }^{13}$ The mammalian clavicle is believed to be the sole exoskeletal remnant in the pectoral girdle ${ }^{14}$ (once braced by an arch of dermal elements and, in fact, connected to the skull in early fishes, the pectoral girdle is now dominated by endoskeletal elements in modern tetrapods). A uniform intramembranous pattern of ossification, as in the avian equivalent - the furcula - would therefore appear plausible. However, the mammalian 
clavicle is a unique amalgamation, developing from two centres ${ }^{15}$ : the medial part ossifies endochondrally, while the lateral part ossifies intramembranously, the two segments being separated by a growth plate. The tapered lateral ends of the clavicles in the present PFMCCD family indicate that correct dosage of MSX2 (as well as CBFA1) is important for intramembranous ossification in the clavicle. However, our observation of additional modelling defects of the central and medial clavicular elements (Figure 2) indicates either a coexisting problem with endochondral ossification, or that in humans, the dichotomy in the developmental origins of the medial and lateral parts of the clavicles is not clearcut. In mice, a generalised deficiency of endochondral ossification was observed only in homozygous null Msx2 mutants. $^{16}$

Both the clinical observations and the molecular analysis support a modification of the current designation of PFMCCD: PFM with clavicular hypoplasia (PFMCH), the term used by Golabi et al, ${ }^{12}$ would be a better descriptor. Given the mild clavicular involvement in our family, which was difficult to assess on physical examination, this finding may be more commonly associated with PFM than currently realised, and may possibly be characteristic of individuals with MSX2 rather than ALX4 mutations. A more careful examination of the clavicles in cases with apparently isolated PFM, and molecular analysis of other families diagnosed with PFMCH will help to clarify these issues.

\section{Acknowledgements}

We thank the family for their cooperation in this study, and Indira Taylor and Mike Oldridge for their help with the mutation and segregation analyses. This work was supported by the Alexander $S$ Onassis Foundation and Medical Research Council (Studentships to LA Mavrogiannis), and by the Wellcome Trust (Senior Clinical Fellowship to AOM Wilkie).

\section{References}

1 Bartsch O, Wuyts W, Van Hul W et al: Delineation of a contiguous gene syndrome with multiple exostoses, enlarged parietal foramina, craniofacial dysostosis, and mental retardation, caused by deletions in the short arm of chromosome 11. Am I Hum Genet 1996; 58: 734-742.

2 Thompson EM, Baraitser M, Hayward RD: Parietal foramina in Saethre-Chotzen syndrome. J Med Genet 1984; 21: 369-372.

3 Wilkie AOM, Tang Z, Elanko $\mathrm{N}$ et al: Functional haploinsufficiency of the human homeobox gene MSX2 causes defects in skull ossification. Nat Genet 2000; 24: 387-390.

4 Wuyts W, Reardon W, Preis S et al: Identification of mutations in the MSX2 homeobox gene in families affected with foramina parietalia permagna. Hum Mol Genet 2000; 9: 1251-1255.

5 Wuyts W, Cleiren E, Homfray Tet al: The ALX4 homeobox gene is mutated in patients with ossification defects of the skull (foramina parietalia permagna, OMIM 168500). J Med Genet 2000; 37: 916-920.

6 Mavrogiannis LA, Antonopoulou I, Baxova A et al: Haploinsufficiency of the human homeobox gene ALX4 causes skull ossification defects. Nat Genet 2001; 27: 17-18.

7 Mundlos S: Cleidocranial dysplasia: clinical and molecular genetics. J Med Genet 1999; 36: 177-182.

8 Otto F, Kanegane H, Mundlos S: Mutations in the RUNX2 gene in patients with cleidocranial dysplasia. Hum Mutat 2002; 19: 209-216.

9 Winter R, Baraitser M: London Dysmorphology Database. London: Oxford University Press, 2001.

10 Eckstein HB, Hoare RD: Congenital parietal foramina associated with faulty ossification of the clavicles. Br J Radiol 1963; 36: 220-221.

11 Hall BD: Syndromes and situations associated with congenital clavicular hypoplasia or agenesis. Prog Clin Biol Res 1982; 104: 279-288.

12 Golabi M, Carey J, Hall BD: Parietal foramina clavicular hypoplasia. An autosomal dominant syndrome. Am J Dis Child 1984; 138: 596-599.

13 Morriss-Kay GM: Derivation of the mammalian skull vault. J Anat 2001; 199: 143-151.

14 Hall BK: Development of the clavicles in birds and mammals. J Exp Zool 2001; 289: 153-161.

15 Huang LF, Fukai N, Selby PB, Olsen BR, Mundlos S: Mouse clavicular development: analysis of wild-type and cleidocranial dysplasia mutant mice. Dev Dyn 1997; 210: 33-40.

16 Satokata I, Ma L, Ohshima H et al: Msx2 deficiency in mice causes pleiotropic defects in bone growth and ectodermal organ formation. Nat Genet 2000; 24: 391-395. 